\title{
Author Correction: Sonic hedgehog accelerates DNA replication to cause replication stress promoting cancer initiation in medulloblastoma
}

Lukas Tamayo-Orrego (D), David Gallo, Frédéric Racicot, Amandine Bemmo, Sushmetha Mohan, Brandon Ho, Samer Salameh, Trang Hoang, Andrew P. Jackson, Grant W. Brown (1) and Frédéric Charron (D)

Correction to: Nature Cancer https://doi.org/10.1038/s43018-020-0094-7, published online 20 July 2020.

In the version of this article initially published, a grant (Canadian Cancer Society Research Institute Innovation grant 705366 to T.H.) was omitted from the Acknowledgements section. The correct section is as follows: “...the Canadian Cancer Society Research Institute (Impact grant 702310 to G.W.B. and Innovation grant 705366 to T.H.).... The error has been corrected in the HTML and PDF versions of the article.

Published online: 19 April 2021

https://doi.org/10.1038/s43018-021-00205-9

(c) The Author(s), under exclusive licence to Springer Nature America, Inc. 2021 